\title{
Lactato sanguíneo em atletas de judô: relato da experiência de coleta durante combates sucessivos em uma competição oficial*
}

\author{
Alexandre Velí Nunes, Rudnei de Andrade, Cláudio R.E. Paiva e Ulf Georg Klemt
}

\section{RESUMO}

Este artigo é um relato de experiência que faz parte de uma investigação sobre a avaliação de atletas de judô de elite. Seu objetivo é comunicar à comunidade científica a metodologia utilizada, assim como descrever os procedimentos e as dificuldades encontradas na execução dessa metodologia. Nessa experiência, foram feitas 155 coletas de lactato sanguíneo em atletas de judô que participaram de uma competição internacional realizada em Porto Alegre, em julho de 1997. Os atletas da seleção gaúcha de judô, previamente selecionados $(\mathrm{n}=21)$, submeteram-se à coleta de sangue do lóbulo da orelha antes e logo após os combates. Além desses, todos os atletas que participaram das lutas finais das respectivas categorias foram incentivados a submeter-se à coleta ao final do combate. Também foi verificada a FC dos atletas através de palpação radial em 15s, no momento da coleta de sangue. Utilizou-se o analisador de lactato portátil Accusport $^{\mathrm{TM}}$ (Boehringer Mannheim Corp., Mannheim, Alemanha) com Test Strips BM Lactate e caneta com microlancetas descartáveis da mesma marca. Constatou-se que a utilização da metodologia e do equipamento acima descritos é apropriada para esse tipo de população e situação, e que os resultados encontrados podem contribuir para a avaliação da intensidade do esforço desses atletas.

Palavras-chave: Judô. Lactato. Avaliação. Atletas. Competição.

\footnotetext{
* Laboratório de Pesquisa do Exercício da Escola Superior de Educação Física da Universidade Federal do Rio Grande do Sul, Porto Alegre, RS
}

Endereço para correspondência:

Alexandre Velí Nunes

Rua Jacinto Osório, 150, apto. 102

90040-290 - Porto Alegre, RS

Tel/Fax: (051) 221-2675

E-mail: Nunes@compuserv.com.br

\begin{abstract}
Blood lactate in judo athletes: report of an experiment of sampling during successive fights in an official competition
\end{abstract}

This article reports an experience that is part of an investigation about elite judo athletes. The purpose of this study is to communicate, to the scientific community, the methodology employed, to describe the procedures and difficulties faced to carry out this methodology. In this experiment, 155 blood lactate samples were made in judo athletes who participated in an international competition that took place Porto Alegre, in July, 1997. The athletes, who were of the judo team from Porto Alegre, and previously selected, $n=21$, were submitted to blood samples taken from the ear lobule before and after the fights. In addition, all athletes participating in the final fights of the respective categories were encouraged to undergo a blood sample at the end of the fight. The athletes' heart rate was verified by radial palpation in $15 \mathrm{~s}$, at the moment the blood was sampled. The portable lactate analyzer Accusport ${ }^{T M}$ (Boehringer Mannheim Corp., Mannheim, Germany) was used together with Test Strips BM Lactate and disposable micro-lancet pens of the same brand. The authors concluded that the use of the methodology and equipment described above are appropriate for this kind of population and circumstance, and that the results obtained may contribute to evaluate the athletes' effort intensity.

Key words: Judo. Lactate. Assessment. Athletes. Competition.

\section{INTRODUÇÃO}

A avaliação de atletas é um tema bastante estudado na medicina do esporte, independentemente da modalidade desportiva. Alguns autores ${ }^{1-3}$ entendem que é necessário avaliar atletas utilizando-se ergômetros mais específicos e que reproduzam o(s) gesto(s) ou a ação aproximada daqueles verificados na competição. Aparentemente, isso não é fácil quando se refere a esportes de combate como o judô. Esta é uma 
atividade que apresenta muitas dificuldades para que sejam reproduzidas em laboratório as ações próprias dos combates. $\mathrm{O}$ objetivo deste relato de experiência é submeter à crítica especializada a metodologia e os procedimentos utilizados neste estudo, e estimular outros pesquisadores a continuarem buscando alternativas para avaliação desse tipo de população.

No judô, os combates desenvolvem-se dentro do tempo regulamentar, cinco minutos para o naipe masculino e quatro minutos para o feminino ${ }^{4}$. No entanto, podem encerrar prematuramente por Ippon $^{(1)}$, além da possibilidade prevista no regulamento de ocorrerem interrupções constantes e variadas. Durante um combate, o tempo pode variar de cinco segundos até mais de cinco minutos, se somadas as pausas intracombates, em média 1'41" 5 . Aproximadamente $50 \%$ das lutas terminam antes do tempo regulamentar ${ }^{6}$, mais exatamente $42 \%$, conforme Castarlenas e Planas. A intensidade do esforço no combate está diretamente relacionada a dois fatores de difícil controle: a tática adotada pelo lutador e o ritmo de luta imposto pelo adversário ${ }^{7,8}$. Além disso, avaliar atletas em uma situação real de competição sempre acarreta dificuldades de ordem burocrática, técnica e metodológica, já que pode influir diretamente na sua performance ${ }^{9-11}$.

Os japoneses foram os primeiros a investigar atletas de judô (décadas de 50 e 60). Basicamente, esses estudos ${ }^{12-17}$ analisavam o comportamento da frequiência cardíaca, avaliações cineantropométricas e medidas de força em determinados grupos musculares, todos estes publicados nos boletins da associação para estudos científicos sobre o judô da Kodokan $^{2}$.

Nos últimos dez anos, muitos pesquisadores têm utilizado o lactato sanguíneo como uma das formas de avaliar os judocas, tanto em situação de combate como em treinamento ${ }^{10,18-}$ 27. Parece que a análise das alterações na concentração do lactato no sangue permite observação mais acurada das reações fisiológicas que as alterações na freqüência cardíaca, para praticantes de judô em treinamento e competição ${ }^{24,29,30}$. A falta de correlação entre a frequiência cardíaca, os parâmetros do balanço ácido-base e a concentração de ácido láctico no sangue, durante atividades com predominância anaeróbica, sugere que o ritmo cardíaco não reflete a intensidade específica da carga de trabalho no judô ${ }^{24,29}$.

\section{RELATO DA EXPERIÊNCIA}

Um estudo feito por pesquisadores do Laboratório de Pesquisa do Exercício (Lapex) da UFRGS, em 1997, avaliou os atletas da seleção gaúcha de judô, inicialmente em laborató- rio e posteriormente durante uma competição. Apresentaremos aqui alguns dados referentes às avaliações feitas em competição, bem como a metodologia e equipamentos utilizados.

Foram dosadas as concentrações de lactato sanguíneo de 21 atletas de judô das categorias juvenil júnior e sênior (maiores de 15 anos), antes e logo após cada combate de determinada competição. Esses atletas compõem a população de um estudo em desenvolvimento na Escola de Educação Física da UFRGS, denominado Avaliação de Atletas de Judô de Alto Rendimento. Todos os atletas envolvidos nesse estudo assinaram um termo de consentimento e as entidades envolvidas na organização da competição receberam informações detalhadas sobre os procedimentos a serem utilizados durante a investigação. O estudo contou com o apoio da Federação Gaúcha de Judô (FGJ) e da Sociedade Ginástica Porto Alegre (Sogipa), organizadores do evento.

Além desses, 25 atletas que participaram das finais desse evento também foram avaliados ao término do combate. Por solicitação do presidente da Federação de Judô do Rio de Janeiro, dois atletas desse Estado que representaram o Brasil nos Jogos Olímpicos de Atlanta foram avaliados em todos os seus combates. No total foram realizadas 155 coletas sem nenhuma perda por problemas de domínio da técnica. Apenas seis amostras deixaram de ser coletadas por dificuldades geradas pela dinâmica da competição. Nas competições oficiais, os atletas dirigem-se diretamente para a área de combate após a chamada ${ }^{3}$. Apesar de estarem orientados a dirigir-se ao local de coleta logo após a primeira chamada, em seis ocasiões o atleta iniciou o combate sem fazê-la.

A coleta dos dados foi realizada durante o 1 은 Troféu Conesul de Judô, competição internacional organizada pela FGJ/ Sogipa. O evento realizou-se em Porto Alegre, de 11 a 13 de julho de 1997, no ginásio de esportes da Sogipa.

A equipe envolvida nesse trabalho era composta de 20 voluntários, alunos do Curso de Mestrado em Ciências do Movimento Humano da ESEF-UFRGS, do curso de graduação e praticantes de judô da equipe da UFRGS. Esse grupo foi subdividido em dois, por função a ser exercida. $O$ primeiro grupo, formado por graduandos da ESEF e judocas, foi treinado para exercer a função de escoltas (total de 17 pessoas); e o segundo grupo, constituído por três alunos do curso de mestrado, ficou responsável pela coleta de sangue, leitura dos resultados e comunicação destes.

Os escoltas foram previamente treinados para as seguintes funções: identificar e acompanhar os atletas selecionados até o local da coleta; verificar a frequiência cardíaca dos atletas e anotar esses resultados na ficha elaborada para esse fim, an-

(1) Pontuação no judô que equivale a um ponto completo e que encerra a luta antes do tempo total previsto.

(2) Kodokan - Fundação nacional japonesa que se originou na primeira escola de judô, criada em 1882.

(3) Os atletas são chamados até três vezes; depois da última chamada o atleta presente é declarado vencedor do combate. 
tes e imediatamente após cada luta; registrar a hora de início e término de cada combate; anotar os resultados da dosagem de lactato que lhes eram comunicados no momento da leitura. A frequiência cardíaca foi verificada através de palpação radial, tomada em 15 segundos, antes de iniciar o combate e assim que os atletas deixavam o tatame ${ }^{(4}$.

Os atletas foram informados pelos escoltas, antes do ingresso no local de competição, onde seria realizada a coleta e qual seria o procedimento. Antes do início da competição a equipe de trabalho reuniu-se para os acertos finais dos procedimentos a serem realizados e também para cientificaremse das determinações dos organizadores do evento.

A dosagem do lactato foi realizada em uma sala situada dentro do ginásio, de frente e bastante próxima da área de competição, localizada logo atrás da mesa central que fazia a chamada dos atletas para os combates. A proximidade viabilizou as coletas logo ao término de cada luta, bem como possibilitou o conhecimento antecipado da ordem dos combates, o que facilitou a localização dos atletas antes mesmo de eles serem chamados para o próximo combate. Foram montados dois pontos de coleta do sangue para análise em lactímetro modelo Accusport TM Portable Lactate Analyser (Boehringer Mannheim Corp., Mannheim, Alemanha), utilizou-se microlanceta descartável para caneta de mesmo modelo com regulagem de 1 a 6 . Utilizou-se sempre a regulagem de nㅇ 6, que permite perfuração de maior profundidade, necessária para a utilização na orelha, conforme projeto-piloto anteriormente realizado. Optou-se por coletar o sangue do lóbulo da orelha, para diminuir o prejuízo na pegada dos atletas durante a competição e minimizar a sensação de dor, que é bem mais pronunciada quando a coleta é feita na ponta dos dedos. Utilizaram-se compressas de gaze hidrófila embebidas em álcool para assepsia do local. Imediatamente após a coleta, pressionava-se o lóbulo da orelha com gaze em solução de epinefrina ${ }^{30}$. Finalmente, o atleta era liberado com o lóbulo da orelha protegido por fita hipoalergênica Micropore $^{\mathrm{TM}}$ da $3 \mathrm{M}$. Essa técnica mostrou-se eficiente para evitar processos hemorrágicos durante os combates subseqüentes. Em apenas dois casos, encontrou-se alguma dificuldade em estancar o sangue após a coleta, ambas sem repercussão para o próximo combate do atleta.

\section{CONCLUSÃO}

Uma das dificuldades em pesquisas que envolvem avaliação de atletas é a transferência dos conhecimentos obtidos em laboratório para situações práticas, principalmente as de competições, quase sempre muito diferentes das condições

(4) Termo de origem japonesa; refere-se a esteiras de palha de arroz de aproximadamente 2 x 1m; atualmente utiliza-se para referir-se à área de combate em uma competição de judô. A terminologia adequada seria shiaijo. de laboratório. Poucos pesquisadores realizam seus estudos em situação de competição, possivelmente porque nestas condições as dificuldades de controle das variáveis apresentam-se sensivelmente aumentadas. Não encontramos um número significativo de estudos desse tipo realizados no Brasil, especialmente se considerarmos o grande número de praticantes e os expressivos resultados do judô nacional.

Nosso estudo caracterizou-se por testar uma metodologia que contemplasse um bom controle de variáveis e uma coleta de dados proveniente de uma situação real de competição. Os resultados obtidos indicam a viabilidade desse procedimento, incentivando outros pesquisadores em ciências do esporte a realizarem estudos utilizando metodologias semelhantes, tanto em atletas de judô, como nos demais esportes de combate.

Destacaremos a seguir alguns aspectos que consideramos importantes no decorrer desta experiência: 1) os escoltas devem ser detalhadamente orientados sobre os procedimentos que deverão realizar; 2) ter experiência em eventos na modalidade; isso mostrou-se importante pela dinâmica deste tipo de competição; 3) a área de coleta deve estar localizada muito próxima da área de competição, com possibilidade de visibilização dos combates; 4) o local da coleta deve proporcionar relativo conforto para os atletas e privacidade.

Nas coletas realizadas antes das lutas, alguns atletas apresentaram, aparentemente, níveis de ansiedade muito elevados e solicitavam que o ato fosse rápido. Ao final dos combates alguns atletas apresentavam alto grau de sudorese, o que dificultava o procedimento de coleta. Finalmente, constatou-se que a interferência dos pesquisadores no andamento do evento deve ser minimizada ao máximo, para não prejudicar o desempenho dos atletas e/ou a organização da competição.

Os procedimentos e o equipamento utilizados mostraramse adequados e de fácil manejo. Esta experiência é parte de um estudo de avaliação de atletas de judô, cujos resultados indicam que a metodologia aplicada pode ser útil para os treinadores e preparadores físicos de esportes de combate, na avaliação da intensidade de esforço dos atletas durante uma competição.

Ao encerrarmos esta comunicação gostaríamos de agradecer aos dirigentes da FGJ e da Sogipa pelo apoio e cedência do local para essa coleta dos dados e muito especialmente aos técnicos e atletas que participaram deste estudo, assim como aos voluntários que executaram suas funções com extrema competência e boa vontade.

\section{REFERÊNCIAS}

1. Baboghluian M, Sencini AC, Mahseredjian F, Barros Neto T. Âmb Med Desp 1996;18:5-13.

2. Pinto Ribeiro J. Limiares metabólicos e ventilatórios durante o exercício: aspectos fisiológicos e metodológicos. Arq Bras Cardiol 1995;2: 171-81. 
3. Sharp N, Koutedakis Y. Anaerobic power and capacity measurements of the upper body in elite judo players, gymnasts and rowers. Aust J Sci Med Sport 1987;3:9-13.

4. Handbook of the International Judo Federation, Judo. Seoul, 1992.

5. Castarlenas JL, Planas A. Estudio de la estructura temporal del combate de judo. Apunts: educación física y deportes 1997;47:32-9.

6. Sikorski W, Michiewicz G, Majle B, Laksa CZ. Estrutura do combate e capacidade de trabalho de judoca. Federação Portuguesa de Judô: Boletim Técnico 1991;1:19-26.

7. Nunes AV. As dificuldades de avaliação de atletas de judô de elite. Revista Perfil 1997;1:13-23.

8. Roquete J, Monteiro L. Avaliação da aptidão física do judoca: aplicação de uma bateria de testes motores aos diferentes escalões etários. Federação Portuguesa de Judô: Boletim Técnico 1991;1:4-18.

9. Gorostiaga EM. Coste energético del combate de judo. Apunts, Med de l'Esport. Barcelona; 97:135-9.

10. Majean H, Gaillat L. Étude de l'acide lactique sanguin chez le judoca au cours de la saison 1984-1985. Médicine du Sport 1986;4:198-203.

11. Silva M. Caracterização do esforço em modalidades desportivas mensuráveis: o judô como caso exemplar. Revista Treino Desportivo 1988; $10: 36-46$

12. Matsumoto Y, Ogawa S, Asami T. Physical fitness of the top judoists in Japan (1967). Bull Scient Stud Judo, Kodokan. Tokyo; 1969. Report III, 1-12.

13. Matsumoto Y, Ogawa S, Asami T. In Making up the standard physical fitness test of judoists. Bull Scient Stud Judo, Kodokan. Tokyo; 1969. Report III, 13-26.

14. Ikai M, Kaneko M. Changes in heart rate during judo practice. Bull Scient Stud Judo, Kodokan. Tokyo; 1969. Report III, 73-9.

15. Ikai M, Hagas S, Kaneko M. The characteristics of physical fitness of judoists from the viewpoint of respiratory and cardiovascular functions. Bull Scient Stud Judo, Kodokan. Tokyo; 1972. Report IV, 43-52.

16. Ogawa S, Imada Y, Akutsu K, Yoshino S, Sugimoto R, Seki T. Energy metabolism in the fundamental throwing techniques of judo. Bull Scient Stud Judo, Kodokan. Tokyo; 1958. Report I, 71-8.

17. Sasa T, Shinozuca T, Katon N, Matsumoto Y, Ikai M, Asami T, Kaneko M. Physical fitness of the top judoists in Japan (1962). Bull Scient Stud Judo, Kodokan. Tokyo; 1963. Report II, 35-44.
18. Amorim AR. Treinamento intermitente no judô e lactato sanguíneo [monografia]. Rio Claro: Unesp, Instituto de Biociências, 1993.

19. Bracht V, Moreira M, Umeda OY. Efeito de lutas sucessivas sobre o nível de ácido láctico sanguíneo de judocas. Rev Bras Ciênc Esp 1992: 3.

20. Callister R, Callister RJ, Staron RS, Fleck SJ, Tesch P, Dudley GA. Physiological caracteristics of elite judo athletes. Int J Sports Med 1991;2: 196-203.

21. Cavazani RN. Lactato antes e após sucessivos combates de judô [monografia]. Rio Claro: Unesp, Instituto de Biociências, 1991.

22. Jarmoluk P. Lactat-und Katecholaminbestimmungen al mittel zur leistungssteuerung im Judo: eine empirische langzeitstudie an weltklasseathletinnem [tese]. Verlag: Sportwissenschaften und Trainingspraxis. Erlensee, 1989.

23. Majean H, Gaillat L. Étude de l'acide lactique sanguin chez le judoca en fonction des méthodes d'entraînement. Med du Sport 1986;4:194-7.

24. Sikorski W, Mickiewicz G. Avaliação fisiológica dos métodos de treino aplicado ao judô. Federação Portuguesa de Judô: Boletim Técnico 1991; $1: 27-32$.

25. Taylor AW, Brassard L. A physiological profile of the Canadian judo team. J Sports Med Phys Fitness 1981;2:160-4.

26. Thomas Ph, Goubault C, Beau MC. Judocas. Évolution de la lactatémie au cours de randoris successifs. Med du Sport 1990;5:234-6.

27. Vidalin H, Dubreuil C, Coudert J. Judokas ceinture noire. Suivi physiologique: etudes biometrique et bio-energetique, Suivi de l'entreinament. Med du Sport 1988;4:184-9.

28. Lukaszewska J, Mickiewicz G, Markowska L, Kakietek M, Zieniawa R, Rzepkiewicz M. Diagnostyka zdolnósci wysilkowej judoków. Sport Wyczynowy 1988;9:33-40.

29. Araújo CGS. Curso - Utilização do Cardiotacômetro de Pulso. Palestra proferida durante o XVII Congresso Pan-americano de Medicina do Esporte; 1997, maio 9; Gramado, Rio Grande do Sul: Confederação Pan-americana de Medicina Desportiva, 1997.

30. Herrera JB. Curso - Lactato. Palestra proferida durante o XVII Congresso Pan-americano de Medicina do Esporte; 1997, maio 8; Gramado, Rio Grande do Sul: Confederação Pan-americana de Medicina Desportiva, 1997. 